\title{
ADVERSE EFFECT PROFILE OF TENOFOVIR DISOPROXIL FUMARATE IN HIV POSITIVE PATIENTS - A PROSPECTIVE STUDY
}

\author{
Andis C. Joseph 1 , K. B. Sanalkumar², C. S. Bharathan'3 , M. A. Andrews ${ }^{4}$, K. Ajithkumar ${ }^{5}$ \\ ${ }^{1}$ Senior Resident, Department of Pharmacology, Government Medical College, Thrissur, Kerala. \\ ${ }^{2}$ Additional Professor, Department of Pharmacology, Government Medical College, Thrissur, Kerala. \\ 3Professor, Department of Pharmacology, Government Medical College, Thrissur, Kerala. \\ 4 Professor, Department of Medicine, Government Medical College, Thrissur, Kerala. \\ ${ }^{5}$ Research Dean, Kerala University of Health Sciences, Thrissur, Kerala.
}

\section{ABSTRACT}

\section{BACKGROUND}

Acquired Immunodeficiency Syndrome, a disease of the human immune system caused by the Human Immunodeficiency Virus (HIV) is a major health problem in many parts of the world. The prognosis was dramatically altered with the introduction of antiretroviral drugs in 1987 and Highly Active Antiretroviral Therapy (HAART) in 1996. Despite the absence of a cure, the natural history of the disease was radically changed since then. This study aimed at documenting and analysing the adverse effect profile of Tenofovir Disoproxil Fumarate-based Antiretroviral Therapy (ART) in HIV positive patients attending Antiretroviral Therapy Centre, Government Medical College, Thrissur.

\section{METHODOLOGY}

HIV positive patients on Tenofovir Disoproxil Fumarate-based antiretroviral treatment attending Antiretroviral Therapy Centre, Government Medical College, Thrissur, were enrolled in the prospective cohort study conducted over 18 months from Jan 2013June 2014. The patients were followed up for 12 months pattern of adverse drug reactions.

\section{RESULTS}

A total of 178 Adverse Drug Reactions (ADRs) were identified, out of which 86 (48.31\%) were in male and 92 (51.68\%) were in female patients. Out of 178 suspected ADRs, 172 (96.6\%) ADRs were reported from outpatient departments. Six patients (3.3\%) required hospital admission. The most commonly identified adverse drug reactions were nausea and vomiting in 54 (30.3\%) patients, headache and fatigue in 30 (16.85\%), heartburn and diarrhoea in 17 (9.5\%) cases, lab abnormalities like dyslipidaemia in 12 (6.74\%), hyperphosphatemia in 10 (5.6\%), hypocalcemia in $5(2.85 \%)$ patients. Out of 178 ADRs, 4 (2.2\%) were of acute renal failure seen. One among the four died even after discontinuation of the drug, 2 were changed to non-Tenofovir based regimen who improved later within 2 months.

\section{CONCLUSION}

In this study of Tenofovir based regimen, the side effects which necessitated regimen change were very few. Majority of the side effects were GI related. The incidence of renal adverse effects were low and were seen in only 4 (2.2\%) patients, out of which one patient died following acute renal failure which could probably be due to Tenofovir. Though renal toxicity of Tenofovir is rare, it can be fatal. So during the course of the treatment, patients should be monitored for nephrotoxicity by laboratory monitoring.

\section{KEYWORDS}

Adverse Effect Profile, Tenofovir, HIV.

HOW TO CITE THIS ARTICLE: Joseph AC, Sanalkumar KB, Bharathan CS, et al. Adverse effect profile of tenofovir disoproxil fumarate in HIV positive patients - a prospective study. J. Evolution Med. Dent. Sci. 2016;5(31):1659-1662, DOI: $10.14260 /$ jemds/2016/391

\section{INTRODUCTION \\ Acquired Immunodeficiency Syndrome is a disease of the human immune system caused by the Human Immunodeficiency Virus (HIV). ${ }^{1}$ Highly Active Antiretroviral Therapy (HAART) introduced in 1996, a combination of agents from 2 different classes of Antiretroviral Agents slows the progression of the disease. ${ }^{2}$}

Financial or Other, Competing Interest: None.

Submission 26-02-2016, Peer Review 25-03-2016,

Acceptance 28-03-2016, Published 18-04-2016.

Corresponding Author:

Dr. K. B. Sanalkumar

Additional Professor,

Department of Pharmacology,

Government Medical College,

Thrissur-680596,

Kerala.

E-mail: sanaldr2001@yahoo.com

DOI: $10.14260 /$ jemds/2016/391
This has dramatically changed to prognosis of the HIV infection. One of the most commonly used drug among them for the first line therapy is Tenofovir Disoproxil Fumarate (TDF). There are many studies on the Adverse Drug Reactions (ADR) due to TDF, but there are only few studies which looked at ADR profile of patients who are receiving treatment in the national public health program of developing countries. Despite the positive therapeutic effects, Antiretroviral Therapy (ART) may cause undesirable adverse effects. Adverse Drug Reactions (ADRs) are one of the reasons for ART interruption among HIV infected patients. ${ }^{3}$

TDF is an antiviral pro-drug acyclic nucleotide diester analog of adenosine monophosphate; the active drug is tenofovir-diphosphate. ${ }^{4}$ It belongs to the class of Nucleotide Reverse Transcriptase Inhibitors. For HIV-1 treatment, TDF is currently recommended as a component of backbone combination in first-line ART. 5 
The most common ADR of TDF are gastrointestinal complaints, headache and asthenia. Tenofovir-associated proximal renal tubulopathy causes excessive renal phosphate and calcium losses and 1-hydroxylation defects of vitamin D. ${ }^{6}$ Signs of proximal renal tubular dysfunction include proteinuria, glucosuria, hypophosphatemia, hypouricemia, hypokalemia, low serum bicarbonate and/or elevated serum creatinine. 7 Tenofovir use has been associated with reduced bone mineral density and increases in biomarkers associated with bone metabolism, which may lead to an increase in fracture risk. ${ }^{8}$ This study was aimed at documenting and analysing the adverse effect profile of Tenofovir Disoproxil Fumarate-based ART in HIV positive patients attending ART Centre, Government Medical College, Thrissur.

\section{METHODOLOGY}

A prospective cohort study was conducted at the ART Centre of Thrissur Medical College, in which two hundred consecutive HIV positive patients on Tenofovir Disoproxil Fumarate-based antiretroviral treatment attending Antiretroviral Therapy Centre, Government Medical College, Thrissur, from Jan 2013 - June 2013 were enrolled in the study after obtaining written informed consent and were followed up for next 12 months up to June 2014. Two regimen were there based on Tenofovir:Tenofovir, Lamivudine and Nevirapine Regimen and Tenofovir, Lamivudine and Efavirenz Regimen. Pregnant and lactating women and those patients who discontinued the drug for more than one month during the study period were excluded from the study. Sample size with $95 \%$ confidence interval was calculated using Statcalc utility of Epi Info Version 6. It was obtained as 197 and for convenience was taken as 200. Though sample size was calculated as 197, a total of 203 patients who were given a regimen based on Tenofovir within the period from Jan 2013June 2013 were included in the study and were followed up for one year; 5 patients with irregular followup/transferred out to other clinics were excluded from the study. So the total number of patients who were evaluated was 198. The 10 patients within the total of 198 patients who died during the course of the study were followed up till their death for any adverse reactions. Ethical clearance for the study was obtained from Institutional Human Ethics Committee of Thrissur Medical College. Information regarding patients' demographics, past medical history, concomitant diseases, family history, occupation and duration of the disease were obtained using a structured proforma. Physical findings and the investigations including complete blood count, liver function test, renal function test, random blood sugar, lipid profile, urine routine examination, serum calcium and phosphate estimation were recorded before starting the treatment and at 4 months, 8 months and 12 months. Ultrasonogram of abdomen was done at the initiation of treatment. ${ }^{6}$ and 12 months and in case of any specific reason/need in between.

The patients were followed up at 2 months interval for clinical examination and the details of adverse events, if any occurred during this period were also recorded in the proforma. The data regarding the presence and absence of subjective side effects were obtained by asking leading questions to the patients or the accompanying person. The causality and severity analysis of the adverse drug reactions were done by using WHO causality assessment scale. ${ }^{9}$ Naranjo's algorithm. ${ }^{10}$ and Hartwig and Seigel scale. ${ }^{11}$ and were recorded. Rechallenge with the same regimen was not attempted due to ethical issues. There were no external sources of funding for the original study, on which the present article is based.

The data was analysed using Epi Info Statistical Package and was presented in two, three and manifold classifications for the comparability of various parameters under the study. Various statistical tests like normal test ( $\mathrm{Z}$ test), Student ' $\mathrm{t}$ ' test, Chi square test, Kolmogorov-Smirnov test and KruskalWallis test were adopted to recognise the patterns of the data and identity. Tests were conducted at $5 \%$ coefficient of significance and inferences were derived.

\section{RESULTS}

We studied 198 HIV positive patients on Tenofovir Disoproxil Fumarate-based antiretroviral treatment attending Antiretroviral Therapy Centre, Government Medical College, Thrissur. Out of these, 185 patients were getting Tenofovir Lamivudine and Nevirapine regimen and 15 patients were under Tenofovir, Lamivudine and Efavirenz regimen. Regarding age about $79 \%$ of patients were in the age group 30-50 yrs. There was no significant difference in the proportions of male and female patients in each age group Males 102 and Females 96.

A total of 178 ADRs were identified, out of which 86 (48.31\%) were in male patients and 92 (51.68\%) female patients. In $55(30.8 \%)$ patients, ADRs noted were abnormal laboratory values. Out of 178 suspected ADRs, 172 (96.6\%) ADRs were reported from outpatient departments. The patients in the age group 41 to 50 years experienced maximum ADRs 60 (33.7\%), followed by 54 (30.33\%) in the age group between 31 and 40 years which were in proportion to the higher number of patients in those age groups. In our study, out of 178 ADRs $6(3.3 \%)$ required hospital admission and gastrointestinal side effects were the commonest ADRs which were more seen in females. Out of 178 ADRs, 4 cases $(2.2 \%)$ of acute renal failure were seen. One of them died even with discontinuation of the drug and treatment in our hospital. ADRs like Fanconi syndrome, hypouricemia, hypokalemia and muscle weakness were not reported.

\begin{tabular}{|c|c|c|c|c|c|c|c|c|c|c|}
\hline \multicolumn{6}{|c|}{ No. of Male Patients } & \multicolumn{5}{|c|}{ No. of Female Patients } \\
\hline $\begin{array}{l}\text { Age in } \\
\text { Years }\end{array}$ & $\begin{array}{l}\text { Abnor- } \\
\text { mal LFT }\end{array}$ & $\begin{array}{l}\text { Abnor- } \\
\text { mal RFT }\end{array}$ & $\begin{array}{c}\text { Abnormal } \\
\text { Lipid Profile }\end{array}$ & $\begin{array}{c}\text { Abnormal Ca 2+ } \\
\text { \& P04 Level }\end{array}$ & $\begin{array}{l}\text { Protei } \\
\text { nuria }\end{array}$ & $\begin{array}{c}\text { Abnorm- } \\
\text { al LFT }\end{array}$ & $\begin{array}{c}\text { Abnor } \\
\text { mal } \\
\text { RFT }\end{array}$ & $\begin{array}{c}\text { Abnormal } \\
\text { Lipid Profile }\end{array}$ & $\begin{array}{l}\text { Abnormal Ca 2+ } \\
\text { \& PO4 Level }\end{array}$ & $\begin{array}{c}\text { Protei- } \\
\text { nuria }\end{array}$ \\
\hline $10--20$ & 1 & 0 & 0 & 1 & 1 & 0 & 0 & 0 & 0 & 0 \\
\hline $21--30$ & 1 & 0 & 1 & 1 & 1 & 1 & 0 & 0 & 2 & 0 \\
\hline $31--40$ & 2 & 1 & 2 & 2 & 1 & 1 & 0 & 2 & 1 & 1 \\
\hline $41--50$ & 3 & 1 & 1 & 2 & 2 & 1 & 2 & 3 & 2 & 2 \\
\hline $\begin{array}{c}\text { Above } \\
50 \\
\end{array}$ & 2 & 0 & 2 & 3 & 1 & 0 & 0 & 1 & 1 & 0 \\
\hline Total & 9 & 2 & 6 & 9 & 6 & 3 & 2 & 6 & 6 & 3 \\
\hline & & & $\begin{array}{r}\text { Tab } \\
\text { Value }\end{array}$ & $\begin{array}{l}\text { nd Gen } \\
\text { ts who }\end{array}$ & Di & $\begin{array}{l}\text { ono } \\
\text { ofov }\end{array}$ & $\begin{array}{r}r n \\
e d\end{array}$ & & & \\
\hline
\end{tabular}


LFT - Liver function test, RFT - Renal function test

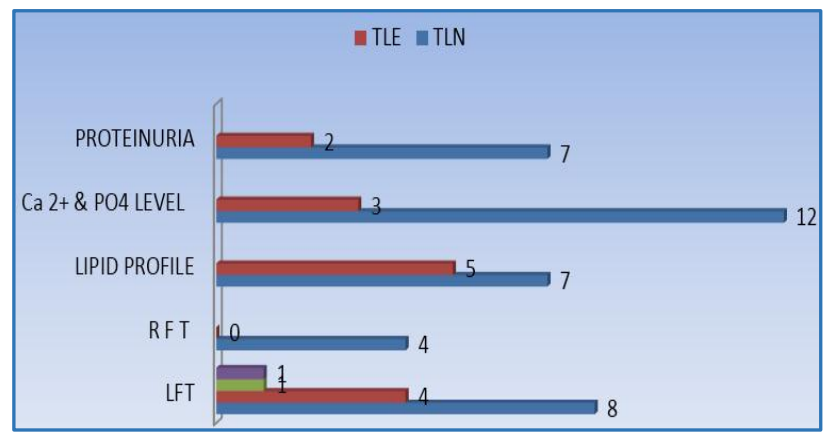

TLN - Tenofovir, Lamivudine and Nevirapine Regimen.

TLE - Tenofovir, Lamivudine and Efavirenz Regimen.

\section{Fig. 1: Distribution of Abnormal Lab Values in Patients who were receiving Tenofovir Based Regimen}

Though only 13 patients were under Tenofovir, Lamivudine and Efavirenz regimen, abnormalities in LFT, Lipid Profile and Serum Ca 2+ and PO4 levels were seen in more proportions in TLE regimen compared to 185 patients with Tenofovir, Lamivudine and Nevirapine regimen. RFT abnormalities noted were elevation in blood urea and serum creatinine levels. Out of 15 patients with abnormalities in serum Ca2+ and P04 level, 11 had hypocalcemia with serum calcium levels ranging from 6.1 to $7.1 \mathrm{mg} / \mathrm{dL}, 2$ patients had hypophosphatemia $(2.1 \mathrm{mg} / \mathrm{dL}$ each $)$ and 2 had hyperphosphatemia ( 4.9 and $5.1 \mathrm{mg} / \mathrm{dL}$ respectively). LFT abnormalities in the form of elevation in liver enzyme levels were seen in 4 out of 13 patients under Tenofovir, Lamivudine and Efavirenz regimen (30.7\%). Lipid profile abnormalities were seen in 5 out of 13 patients (38.4\%) and Ca2+ and PO4 level abnormalities in 3 out of $13(23 \%)$ patients under Tenofovir, Lamivudine and Efavirenz regimen. The results were verified statistically and found to be significant as the $p$ values were $<0.05$.

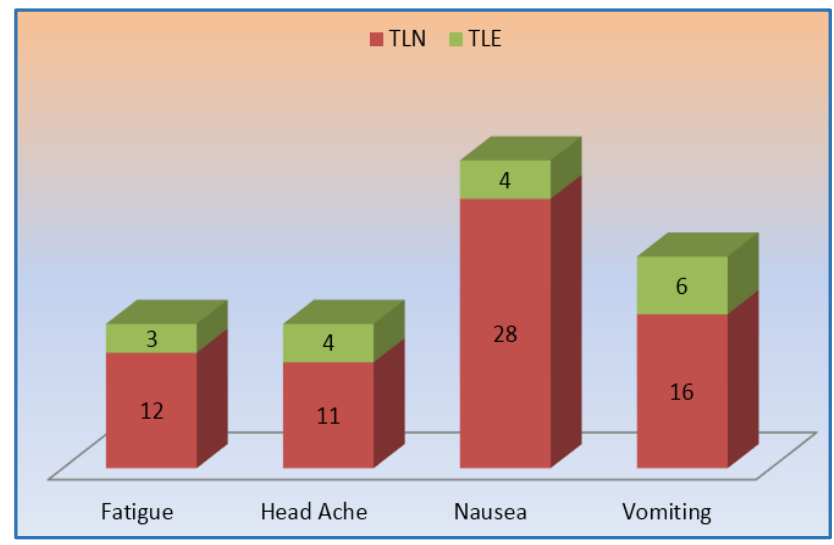

Fig. 2: Distribution of Common Clinical Adverse Events in Patients who were receiving Tenofovir Based Regimen

Common clinical adverse effects were seen in more proportions in Tenofovir, Lamivudine and Efavirenz regimen considering the fact that there are only 13 patients getting that regimen; $23.7 \%$ of patients under Tenofovir, Lamivudine and Efavirenz regimen had fatigue compared to $6.4 \%$ in Tenofovir, Lamivudine and Nevirapine regimen ( $p$ value $=0.02<0.05) ; 30.7 \%$ of patients under Tenofovir Lamivudine and Efavirenz regimen had headache compared to $5.94 \%$ in other regimen ( $\mathrm{p}$ value $=0.0019<0.05$ ); $30.7 \%$ of patients under Tenofovir, Lamivudine and Efavirenz regimen had nausea compared to $16 \%$ in Tenofovir, Lamivudine and Nevirapine regimen ( $p$ value $=0.0003<0.05$ ).

\section{Causality Assessment by Naranjo's Scale}

Causality assessment was done by using both Naranjo's and WHO scale. The assessment by Naranjo's scale showed that out of 178 ADRs 169 (95\%) ADR's were possible, 9 (5\%) were classified as probable. None of them were found out to be definitely related to the drug as drug level estimation in the blood and rechallenge with the same regimen with/without dose adjustments were not attempted due to ethical issues.

\section{WHO Probability Assessment Scale}

The assessment done by using WHO scale revealed that out of 178 ADR's 168 (94.4\%) ADR's were possibly drug-related, 10 (5.6\%) ADRs were probably drug-related and $0(0.0 \%)$ ADR were identified as certain as rechallenge with the same drugs was not attempted due to ethical issues.

\section{Severity Assessment by Modified Hartwig and Siegel Scale}

The severity assessment showed that 95 (53.37\%) ADRs were moderate, 77 (43.25\%) ADRs were mild and 6 (3.3\%) ADRs were severe when the levels of severity of ADRs were assessed using the scale. Lethal effect was observed or produced in 1 patient $(0.5 \%)$.

\section{DISCUSSION}

Tenofovir has emerged as a first line drug for treatment of AIDS. In this study 198 HIV positive patients on Tenofovir Disoproxil Fumarate-based antiretroviral treatment attending ART Centre, Government Medical College, Thrissur, were enrolled and followed up to record and analyse the adverse effects for a period of one year in which Tenofovir was given $300 \mathrm{mg}$ once daily. The important observations were:

The most commonly identified adverse drug reactions were nausea and vomiting in $54(30.3 \%)$ cases, headache and fatigue in $30(16.85 \%)$ cases, heartburn and diarrhoea in 17 (9.5\%) cases, lab abnormalities like dyslipidaemia in 12 (6.74\%), hyperphosphatemia in $10(5.6 \%)$, hypocalcemia in 5 $(2.85 \%)$ and renal problems in $4(2.24 \%)$. In this study acute renal failure was seen only with Tenofovir, Lamivudine and Efavirenz regimen. Common clinical adverse effects were seen in more proportion in Tenofovir, Lamivudine and Efavirenz regimen considering the fact there were only 13 patients getting that regimen. It was verified statistically and incidences of fatigue, headache and vomiting were found statistically significant.

Regimen change due to Drug Toxicity was needed only in $2 \%$ of patients compared to $27 \%$ in a study conducted by Ajith Sivadasan et al. ${ }^{3}$ which may be due to difference in the regimens used in that study, as the initial ART regimens used in that study were Lamivudine (3TC) with Stavudine (d4T) (in 76\%) or Azidothymidine (AZT) and Nevirapine (NVP) (in $86 \%$ ) or Efavirenz (EFV). This may also account for the reduced incidences of adverse effects caused by Tenofovir Disoproxil Fumarate-based antiretroviral treatment. ADRs 
like Fanconi syndrome and Muscle weakness were not reported when compared to a similar study conducted by Shubhanker Mitra et al.12 to describe the incidence and characteristics of Tenofovir (TDF) induced nephrotoxicity.

The prevalence of comorbidities like hypertension, dyslipidaemia and diabetes were lesser when compared to the study by Chu $\mathrm{C}$ et al. ${ }^{13}$ who noticed prevalence rates of $26 \%, 48 \%$ and $13 \%$ for hypertension, dyslipidaemia and diabetes respectively, which may be due to differences in the ethnicity and the higher sample size of 854 studied there. Elevation in liver enzyme levels were seen only in less than $10 \%$ compared to one-year long study of 142 patients on Tenofovir by Sanchez-Conde M et al. ${ }^{14}$ which may be due to the nearly $45 \%$ prevalence of Hepatitis $\mathrm{C}$ in that population. In our entire sample, none of the patients had Hepatitis $C$. The occurrences of nephrolithiasis and hydronephrosis were not reported compared to the study by Cicconi $\mathrm{P}$ et al. 15 in which such adverse events were noted, which may be due to factors other than the treatment and also due to the differences in the dietary habits of South India when compared to the western population.

Due to constraints of facilities, investigation like Bone Densitometry was not done which may be considered as a limitation of the study. As the scientifically calculated sample size was met, the overall interpretation of results can be extrapolated to a higher sample size which shows the generalizability and external validity of the study results. However, our patients might not be completely representative of HIV-infected patients in the country as we have studied only those patients who were followed up at the ART Centre in our medical college. The potential sources of bias were taken into account to reduce the imprecision of the results.

\section{CONCLUSION}

\section{At the end of the study the following conclusions were} derived

Out of the 198 patients, a total of 178 adverse reactions were recorded and analysed, out of which serious adverse events were less. Out of these, gastrointestinal side effects were the highest compared to the other systems. The incidence of renal adverse effects was low and were seen in only 4 patients, out of which one patient died following acute renal failure, which could probably be due to the adverse effect of the drug. So during the course of the treatment, patients should be monitored for nephrotoxicity. Hence, Tenofovir based regimen can be considered as the backbone component in first line Highly Active Antiretroviral Therapy.

\section{REFERENCES}

1. Sepkowitz KA. AIDS-the first 20 years. N Engl J Med 2001;344(23):1764-72.
2. May MT, Ingle SM. Life expectancy of HIV-positive adults: a review. Sexual health 2011;8(4):526-33. doi:10.1071/SH11046. PMID 22127039.

3. Ajith Sivadasan, Abraham OC, Priscilla Rupali, et al. High rates of regimen change due to drug toxicity among a cohort of south indian adults with HIV infection initiated on generic, first-line antiretroviral treatment. JAPI 2009;57:384-8.

4. Thompson MA, Aberg JA, Cahn P, et al. Antiretroviral treatment of adult HIV infection: 2010 recommendations of the international AIDS society-USA panel. JAMA 2010;304(3):321-33.

5. Current HIV treatment guidelines. National Institute of Health (NIH). [internet] Accessed: 27 Feb 2013 Available: http://www.aidsinfo.nih.gov/guidelines/.

6. Havens PL, Kiser JJ, Stephensen CB. Association of higher plasma vitamin D binding protein and lower free calcitriol levels with tenofovir disoproxil fumarate use and plasma and intracellular tenofovir pharmacokinetics: cause of a functional vitamin D deficiency? Antimicrob Agents Chemother 2013;57(11):5619-28.

7. Samir K Gupta. Tenofovir-associated fanconi syndrome: review of the FDA adverse event reporting system. AIDS Patient Care and STDs 2008;22(2):99-103.

8. Kohler J James, Seyed H Hosseini, Elgin Green, et al Tenofovir renal proximal tubular toxicity is regulated by OAT1 and MRP4 transporters. Lab Invest 2011;91(6):852-8.

9. Syed Ahmed Zaki. Adverse drug reaction and causality assessment scales. Lung India 2011;28(2):152-3.

10. Naranjo CA, Busto U, Sellers EM, et al. A method for estimating the probability of adverse drug reactions. Clin Pharmacol Ther 1981;30(2):239-45.

11. Hartwig SC, Siegel J, Schneider PJ. Preventability and severity assessment in reporting adverse drug reactions. Am J Hosp Pharm 1992;49(9):2229-32.

12. Shubhanker Mitra, Rupali Priscilla, Rajeev Karthik, et al. Renal tubular dysfunction associated with tenofovir therapy. JAPI 2014;62(7):580-2.

13. Chu C, Umanski G, Blank A, et al. Comorbidity-related treatment outcomes among HIV-infected adults in the bronx, NY. J Urban Health 2011;88(3):507-16. doi:10.1007/s11524-010-9540-7.

14. Sanchez-Conde M, Gil P, Sánchez-Somolinos $M$, et al. Hepatic and renal safety of tenofovir in HIV-infected patients with hepatitis $\mathrm{C}$, including patients on interferon plus ribavirin. HIV Clin Trials 2005;6(5):27880.

15. Cicconi P, Bongiovanni M, Melzi S, et al. Nephrolithiasis and hydronephrosis in an HIV-infected man receiving tenofovir. Int J Antimicrob Agents 2004;24(3):284-5. 